\title{
Effects of Environmental Enrichment on Nicotine Sensitization in Rats Neonatally Treated with Quinpirole: Analyses of Glial Cell Line-Derived Neurotrophic Factor and Implications towards Schizophrenia
}

\author{
Russell W. Brown ${ }^{a}$ Marjorie A. Schlitt ${ }^{b}$ Alex S. Owens ${ }^{b}$ Caitlynn C. DePreter ${ }^{b}$ \\ Elizabeth D. Cummins ${ }^{b}$ Seth L. Kirby ${ }^{b}$ W. Drew Gilla Katherine C. Burgess ${ }^{a}$ \\ ${ }^{a}$ Department of Biomedical Sciences, James H. Quillen College of Medicine, East Tennessee State University, \\ Johnson City, TN, USA; ${ }^{b}$ Department of Psychology, East Tennessee State University, Johnson City, TN, USA
}

\section{Keywords}

Dopamine - Dopamine receptor - Developing brain ·

Animal model · Neurotrophic factor - Nucleus accumbens ·

Schizophrenia

\begin{abstract}
The current study analyzed the effects of environmental enrichment versus isolation housing on the behavioral sensitization to nicotine in the neonatal quinpirole (NQ; dopamine $D_{2}$-like agonist) model of dopamine $D_{2}$ receptor supersensitivity, a rodent model of schizophrenia. NQ treatment in rats increases dopamine $D_{2}$ receptor sensitivity throughout the animal's lifetime, consistent with schizophrenia. Animals were administered NQ (1 mg/kg) or saline (NS) from postnatal day (P)1 to P21, weaned, and immediately placed into enriched housing or isolated in wire cages throughout the experiment. Rats were behaviorally sensitized to nicotine $(0.5 \mathrm{mg} / \mathrm{kg}$ base) or saline every consecutive day from P38 to $\mathrm{P} 45$, and brain tissue was harvested at P46. Results revealed that neither housing condition reduced nicotine sensitization in NQ rats, whereas enrichment reduced sensitization to nicotine in NS-treated animals. The nucleus accumbens
\end{abstract}

(C) 2018 S. Karger AG, Basel

E-Mail karger@karger.com www.karger.com/dne
(NACc) was analyzed for glial cell line-derived neurotrophic factor (GDNF), a neurotrophin important in dopamine plasticity. Results were complex, and revealed that NAcc GDNF was increased in animals given nicotine, regardless of housing condition. Further, enrichment increased GDNF in NQ rats regardless of adolescent drug treatment and in NS-treated rats given nicotine, but did not increase GDNF in NS-treated controls compared to the isolated housing condition. This study demonstrates that environmental experience has a prominent impact on the behavioral and the neural plasticity NAcc response to nicotine in adolescence.

(c) 2018 S. Karger AG, Basel

\section{Introduction}

In a series of studies, we have developed a preclinical model of psychosis by neonatally treating rats with the dopamine $\mathrm{D}_{2} / \mathrm{D}_{3}$ agonist quinpirole (NQ) from postnatal day $(\mathrm{P}) 1$ to $\mathrm{P} 21$. This treatment results in an increase of sensitivity of dopamine $\mathrm{D}_{2}$-like receptors throughout the animal's lifetime but the $\mathrm{D}_{2}$ receptor number is unaltered [1]. Increases in $\mathrm{D}_{2}$ sensitivity and/or functioning are

Prof. Russell W. Brown

Department of Biomedical Sciences

James H. Quillen College of Medicine, East Tennessee State University

Johnson City, TN 37614 (USA)

E-Mail brown1@etsu.edu 
common in psychosis, especially schizophrenia $[2,3]$. We have shown that NQ treatment in rats leads to enhanced behavioral and dopaminergic response to nicotine [4] as well as to amphetamine $[5,6]$. We have also validated NQ treatment as a model of psychosis in several other behavioral and neurobiological domains [7], meeting all standards of model validity [8]. Important to the present study, individuals diagnosed with schizophrenia are 3-4 times more likely to smoke cigarettes compared to the normal population $[9,10]$, but there is no delineated mechanism for this increase. In the present study, we analyzed the effects of environmental enrichment and isolated rearing on behavioral and glial cell line-derived neurotrophic factor (GDNF) response to nicotine in adolescence, a critical period of vulnerability to the initiation of smoking behavior [11].

Behavioral sensitization to psychostimulants has long been established as a measurement of the combination of learned associations and the arousing aspects of behavioral response to psychostimulant drugs. Animals are administered the drug of interest and placed into a locomotor arena with activity monitored. Typically, administration of the same dose of drug results in a significant increase in activity after each administration over a 1- to 2 -week period [12]. Sensitization to nicotine is unique in that the initial response is hypoactivity, and further administration results in an increase of activity after each subsequent administration. Several studies have shown that increases in dopamine release in the nucleus accumbens (NAcc) mediate this response to nicotine [13] as well as to other psychostimulants [14].

There has been interest in analyzing the effects of experience on the behavioral response to addictive drugs. Manipulating the housing conditions of rodents is one way in which to measure the effects of experience on development and how this may interact with the behavioral response to drugs of abuse. In general, past work has shown that environmental enrichment during adolescence and/or adulthood attenuates sensitization to psychostimulants and reduces escalation of the self-administration of psychostimulant drugs in rats [15-17]. Further, isolated housing results in an enhancement of the response to psychostimulants $[18,19]$.

There has also been work to analyze the effects of enrichment on neural plasticity and neurotrophic factors in the brain. In general, these studies have shown that environmental enrichment results in a significant increase of a host of neurotrophic factors. Specific to this study, increases of brain-derived neurotrophic factor (BDNF) in the hippocampus, basal forebrain, and cerebral cortex have been reported as a result of environmental enrichment $[20,21]$. GDNF is known to play a primary role in dopamine plasticity in brain areas that are known to be important in brain addiction, including the NAcc and dorsal striatum [22]. Interestingly, there has only been one study to demonstrate that environmental enrichment increases GDNF immunoreactivity in the brain, but that study focused on the hippocampus, where GDNF density is relatively low [23].

Very few studies have been published analyzing the effects of environmental enrichment and/or isolation rearing in animal models of psychopathology and their behavioral response to drugs of addiction. Of note, isolation rearing during the adolescent period has been validated in and of itself as a model of schizophrenia in rodents [24]. There are a number of studies performed on isolation rearing in rats, but none have studied its effects on the behavioral or plasticity response to nicotine. The effects of environmental enrichment have revealed the ability to rescue behavioral deficits in mouse models of psychosis $[25,26]$ as well as in the neonatal MK-801 NMDA antagonist model in rats $[27,28]$. More recently, Santos et al. [29] reported that environmental enrichment reduced behavioral deficits in spontaneously hypertensive rats (SHR), as well as increasing neurogenesis as in the CA1 and CA3 regions of the hippocampus of both SHR and controls. However, there was no differential effect due to housing condition across groups. Interestingly, in the polyinosinic-polycytidylic acid (poly I:C) immune activation rodent model of schizophrenia, environmental enrichment did not affect increases in dopamine $\mathrm{D}_{2}$ receptor activation, expression, or amphetamine sensitization, or influence social interaction in this model $[30,31]$. Therefore, the effects of enrichment appear to be equivocal across rodent models of schizophrenia relative to eliminating behavioral deficits, but there have been no studies that have analyzed the effects of enrichment and the behavioral response to nicotine in a rodent model of psychosis. Further, there have not been many studies analyzing the effects of isolation during the adolescent period on the behavioral response to nicotine in any rodent model of schizophrenia. In the present study, we compared the effects of environmental enrichment and isolation rearing in the NQ model and its behavioral response to nicotine. In addition, based on the important role of the NAcc in nicotine behavioral sensitization [32], the NAcc was analyzed for GDNF protein on the day after sensitization was complete. 


\section{Methods}

Subjects

A total of 10 adult timed pregnant female rats were ordered from Envigo, Inc. (Indianapolis, IN, USA) at gestational day 15. Approximately a week later, the litter was born and this was designated as P0. The rats were weaned at P21 from the female dam, and placed into either enriched housing or isolated housing as described below. One male and one female was assigned to each housing/drug condition from each litter to control for within-litter variance. The animals were housed on a 12-h light/dark cycle and food was available ad libitum throughout the experiment. All procedures were approved by the East Tennessee State University Animal Care and Use Committee which is congruent with the NIH Guide for the Care and Use of Animals.

\section{Neonatal Drug Treatment}

The animals were given a single daily intraperitoneal injection of either quinpirole (NQ; $1 \mathrm{mg} / \mathrm{kg}$ ) or saline from P1 to P21. The rationale for NQ treatment from P1 to P21 is that this treatment has been shown to result in an increase of dopamine $\mathrm{D}_{2}$ receptor sensitivity throughout the animal's lifetime [1]. Further, NQ treatment results in decreased expression of regulator of G-protein signaling 9 (RGS9) in dopamine terminal areas of the brain [33]. RGS9 is involved in the regulation of G-protein signaling at the dopamine $\mathrm{D}_{2}$ receptor [34], and decreased expression of RGS9 is consistent with postmortem data in individuals diagnosed with schizophrenia [2]. After the animals were weaned at P21, they were randomly divided and placed into isolation or environmentally enriched housing and remained in these housing conditions throughout behavioral testing until brain tissue was harvested at $\mathrm{P} 46$. The rationale for the choice of enrichment only during the adolescent period was to analyze the effects of environmental enrichment/isolation during a critical period of dopaminergic development [35]. In addition, adolescence is a period when smoking behavior often initiates.

\section{Housing Conditions}

Animals housed in isolation were singly housed in stainless steel wire hanging cages $(40.6 \mathrm{~cm}$ width $\times 17.8 \mathrm{~cm}$ height $)$ with a wire mesh floor and no objects were placed into the cage. Animals housed in the enriched condition were socially housed in a large wire cage with a solid stainless steel metal floor covered in bedding (76.2 $\mathrm{cm}$ width $\times 91 \mathrm{~cm}$ height) with at least 6 other rats, and the animals were always of the same sex when housed together in enrichment. The enriched condition contained several different objects animals could interact with, and half of these objects were replaced every 2 days and rotated into new positions daily for the entire study. The animals in the environmentally enriched condition were also handled daily by an experimenter, whereas the animals in isolation were not handled until behavioral testing began (outside of cage changes by animal care staff).

\section{Adolescent Drug Treatment and Behavioral Sensitization}

Behavioral testing began on P35. The animals were removed from their enriched or isolated housing conditions and placed into square locomotor Plexiglas arenas $(30 \mathrm{~cm} /$ side $)$ for 3 consecutive days from P35 to P37 for habituation, and began drug treatment on P38. For habituation, the animals were administered saline intraperitoneally and placed into the locomotor arena $10 \mathrm{~min}$ later; the distance traveled (in meters) was recorded for $10 \mathrm{~min}$ using
AnyMaze software (Stoelting Co., Wood Dale, IL, USA). After habituation was complete, nicotine treatment began. Beginning on P38, the animals were given an intraperitoneal injection of either nicotine $(0.5 \mathrm{mg} / \mathrm{kg}$ free base $)$ or saline. This dose was used because we have demonstrated in past work that NQ rats demonstrate enhanced sensitization to this dose of nicotine. Approximately 10 min after the injection, all animals were placed into the locomotor arenas and the distance traveled was recorded using AnyMaze software for a total of $10 \mathrm{~min}$. After behavioral testing, the animals were returned to their respective housing conditions; the animals were never outside of their housing conditions for more than $1 \mathrm{~h}$ during behavioral testing. There were a total of 7-10 animals per group. The animals were administered saline or nicotine for 8 consecutive days from P38 to P45, and brain tissue was harvested $24 \mathrm{~h}$ after the last drug administration on $\mathrm{P} 46$. The rationale for studying animals during adolescence was that this developmental period has been identified as particularly vulnerable in individuals diagnosed with schizophrenia [36].

\section{GDNF ELISA}

For the GDNF ELISA, the NAcc was micropunched away from the rest of the brain tissue and analyzed using the Emax immunoassay system (Promega, Madison, WI, USA). The antibodies used in this system have been shown to have $<3 \%$ cross-reactivity with other neurotrophic factors at $10 \mathrm{ng} / \mathrm{mL}$ [37]. All brain tissue samples were weighed and homogenized using a Fisher Scientific sonic dismembrator 500 in $250 \mu \mathrm{L}$ of RIPA cell lysis buffer $(150 \mathrm{mM}$ $\mathrm{NaCl}, 50 \mathrm{~mm}$ Tris- $\mathrm{HCl}, 1.0 \% \mathrm{NP}-40,0.5 \%$ sodium deoxycholate, and $0.1 \%$ sodium dodecyl sulfate), which included several protease and phosphatase inhibitors (P5726, P8340, and P0044; Sigma-Aldrich, St. Louis, MO, USA). The brain region and RIPA buffer was centrifuged at $20,000 \mathrm{~g}$ for $25 \mathrm{~min}$ at $4{ }^{\circ} \mathrm{C}$. A 96-well plate was coated using an anti-GDNF polyclonal antibody mixed into a carbonate coating buffer $(\mathrm{pH}=8.2)$ and incubated at $4{ }^{\circ} \mathrm{C}$ for $24 \mathrm{~h}$. The following day, any nonspecific binding was blocked using a block and sample buffer provided by the manufacturer. Approximately $1 \mathrm{~h}$ later, the samples were applied to the plate, followed 6 $\mathrm{h}$ later by an anti-human GDNF monoclonal antibody and incubated for $24 \mathrm{~h}$. At this point, the anti-chicken IgY horseradish peroxidase conjugate was added, followed $1 \mathrm{~h}$ later by the TMB One Solution. The reaction was stopped using $1 \mathrm{~N}$ hydrochloric acid. Optical density was measured using a Bio-Tek ELx800 microplate reader (Winooski, VT, USA).

\section{Research Design}

Factors in the design included neonatal drug treatment (saline or quinpirole), housing condition (enriched or isolated), and adolescent drug treatment (saline or nicotine). The distance traveled (in meters) was measured on each day of behavioral testing. Distance traveled was analyzed as the dependent measure for habituation and for the behavioral response to acute nicotine (or saline). The distance traveled on the first day of sensitization was subtracted from that on the final day of sensitization to determine the behavioral response to subchronic nicotine. Although sex was initially included as a factor, there were no significant main effects or interactions using sex as a factor and it was dropped from the analysis. A two-way ANOVA with neonatal drug treatment and housing condition serving as factors was used for habituation. A threeway ANOVA was utilized for the behavioral response to acute nicotine, for behavioral sensitization, and for analysis of GDNF in the
66

Dev Neurosci 2018;40:64-72

DOI: $10.1159 / 000486391$
Brown/Schlitt/Owens/DePreter/

Cummins/Kirby/Gill/Burgess 
NAcc with neonatal drug treatment, housing condition, and adolescent drug treatment as factors. A Newman-Keuls post hoc test was used to analyze significant interactions. For all figures with the exception of that concerning habituation (Fig. 1a), the initial letter code represents neonatal drug treatment (NS, neonatal saline; NQ, neonatal quinpirole) and the second letter represents adolescent drug treatment ( $\mathrm{S}$, saline, $\mathrm{N}$, nicotine).

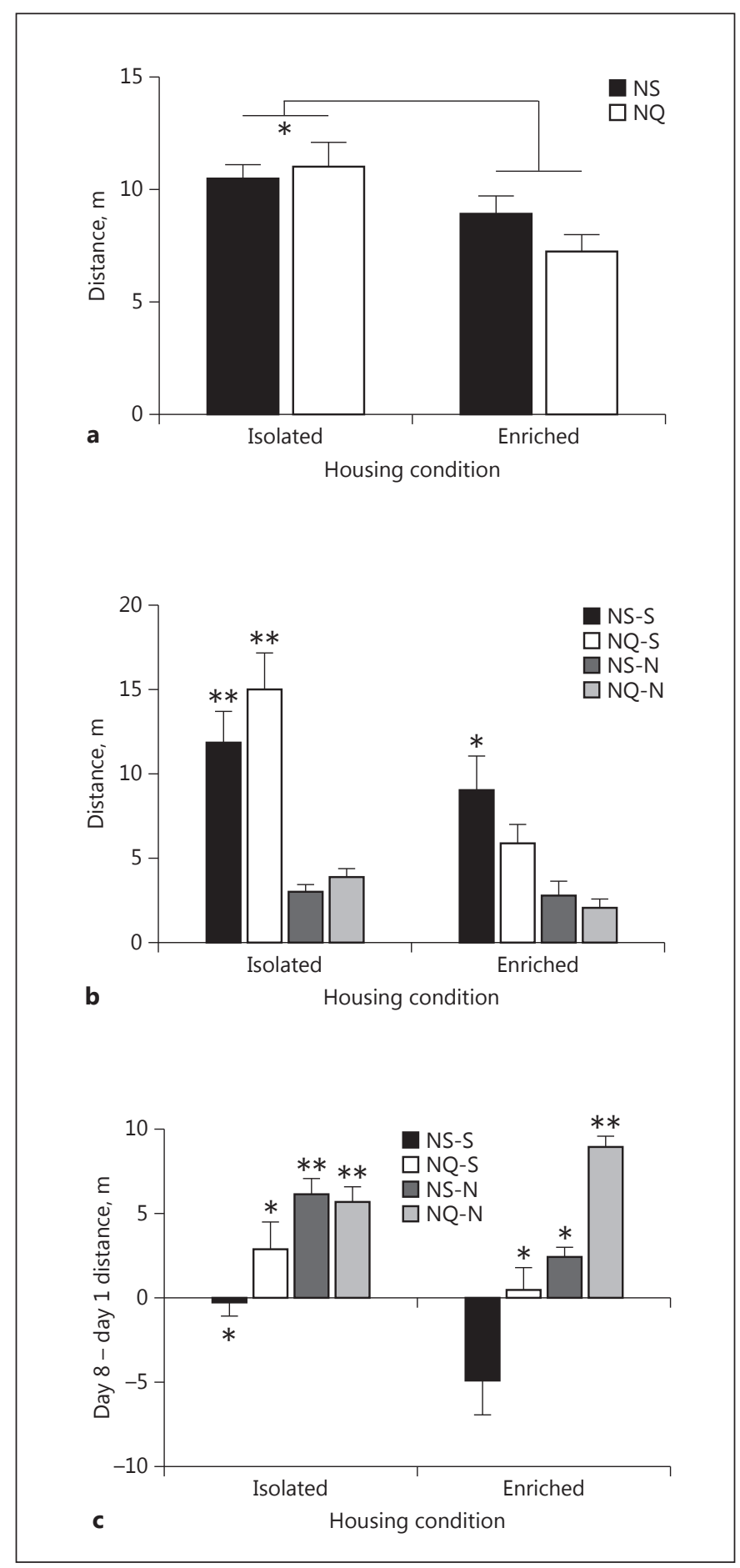

Effects of Environmental Enrichment on Nicotine Sensitization in NQ Rats

\section{Results}

\section{Behavioral Sensitization}

Figure 1a represents distance traveled as a function of neonatal drug treatment and housing condition for the 3 days of habituation (P35-P37). For this analysis, only neonatal drug treatment and housing condition were included as factors, because habituation preceded adolescent drug treatment. A two-way ANOVA of the mean distance traveled revealed a significant main effect of housing condition during habituation: $F(1,66)=8.98$, $p<0.004$. Therefore, isolated housing increased overall baseline activity compared to the enriched housing condition, but this was not altered by neonatal drug treatment.

Figure $1 \mathrm{~b}$ represents distance traveled as a function of housing condition, neonatal drug treatment, and adolescent drug treatment for the acute behavioral response to nicotine on day 1 of drug treatment. A three-way ANOVA revealed significant main effects of housing condition: $F(1,65)=13.06, p<0.001$, and adolescent drug treatment: $F(1,65)=60.26, p<0.001$, and significant two-way interactions of neonatal drug treatment $\times$ housing condition: $F(1,65)=4.23, p<0.04$ and housing condition $\times$ adolescent drug treatment: $F(1,65)=7.57, p<0.008$. Based on these significant interactions, we conducted a NewmanKeuls post hoc test that revealed that isolated conditions NS-S and NQ-S demonstrated equivalent levels of activ-

Fig. 1. a Distance traveled is presented as a function of group for habituation. For all figures, the initial letter code represents neonatal drug treatment (NS, neonatal saline; NQ, neonatal quinpirole) and the second letter represents adolescent drug treatment ( $\mathrm{S}$, saline, $\mathrm{N}$, nicotine). There were no significant differences in distance traveled between groups; thus, housing condition did not appear to affect baseline activity. ${ }^{*} p<0.05$. b Distance traveled is presented as a function of neonatal drug treatment and housing condition for the acute response to nicotine on the first day of adolescent drug treatment. Isolated groups NS-S and NQ-S demonstrated equivalent levels of activity, which were equivalent, and significantly greater than all other groups (indicated by $* * \mathrm{p}<$ $0.05)$. In addition, enriched condition group NS-S had significantly greater activity level than all groups that received nicotine (indicated by $* p<0.05)$. c The difference in distance traveled on day 8 versus day 1 is presented as a function of neonatal drug treatment and housing condition for nicotine behavioral sensitization. Enriched group NQ-N was equivalent to isolated groups NQ-N and NS-N, and these 3 groups demonstrated more robust sensitization to nicotine than all other groups (indicated by ${ }^{* *} p<0.05$ ). Isolated groups NS-S and NQ-S and enriched groups NQ-S and NS-N were all statistically equivalent and mean differences in activity were significantly greater than those in enriched group NS-S (indicated by $*<0.05)$. 
ity, which were equivalent, and significantly greater than all other groups. In addition, enriched condition group NS-S had significantly greater activity level than all groups that received nicotine. Essentially, the analysis of the first day of behavioral sensitization testing demonstrated that nicotine resulted in a hypoactive response regardless of neonatal drug treatment or housing condition, and that animals in the isolated condition administered saline demonstrated increased locomotor activity compared to their enriched counterparts administered saline, supporting the analysis of habituation in Figure 1a.

Figure $1 \mathrm{c}$ represents the difference in distance traveled on day 8 versus day 1 as a function of housing condition, neonatal drug treatment, and adolescent drug treatment. A three-way ANOVA revealed a significant main effect of neonatal drug treatment: $F(1,65)=18.07, p<0.001$, housing condition: $F(1,65)=7.83, p<0.01$, and adolescent drug treatment: $F(1,65)=44.11, p<0.001$, a significant two-way interaction of housing condition $x$ adolescent drug treatment: $F(1,65)=6.67, p<0.01$, and a significant three-way interaction of neonatal drug treatment $\times$ housing condition $\times$ adolescent drug treatment: $F(1,65)=$ $4.64, p<0.03$. Newman-Keuls post hoc analysis revealed that enriched group NQ-N was equivalent to isolated groups NQ-N and NS-N, and these three groups demonstrated more robust sensitization to nicotine than all other groups. In addition, isolated groups NS-S and NQ-S and enriched groups NQ-S and NS-N were all equivalent, and demonstrated significantly increased activity compared to enriched group NS-S, which was significantly lower than all other groups.

GDNF ELISA. Figure 2 represents GDNF as a function of housing condition and drug treatment for the NAcc. A three-way ANOVA revealed a significant main effect of housing condition: $F(1,43)=10.97, p<0.002$, and adolescent drug treatment: $F(1,43)=5.80, p<0.02$, and two significant two-way interactions of neonatal drug treatment $\times$ housing condition: $F(1,43)=12.97, p<0.001$, and neonatal drug treatment $\times$ adolescent drug treatment: $F(1,43)=4.86, p<0.03$. Newman-Keuls post hoc analysis revealed that isolated group NS-N was statistically equivalent to enriched groups NQ-S, NS-N, and NQ-N, and all of these groups demonstrated greater levels of GDNF than all other groups. Therefore, it appears that isolation during adolescence blocked the effects of nicotine on NAcc GDNF in NQ animals, but did not affect NAcc GDNF in NS-treated animals. Further, enrichment increased NAcc GDNF levels in animals that received NQ treatment regardless of adolescent drug treatment, but did not appear to affect the increase in GDNF in NS-treat-

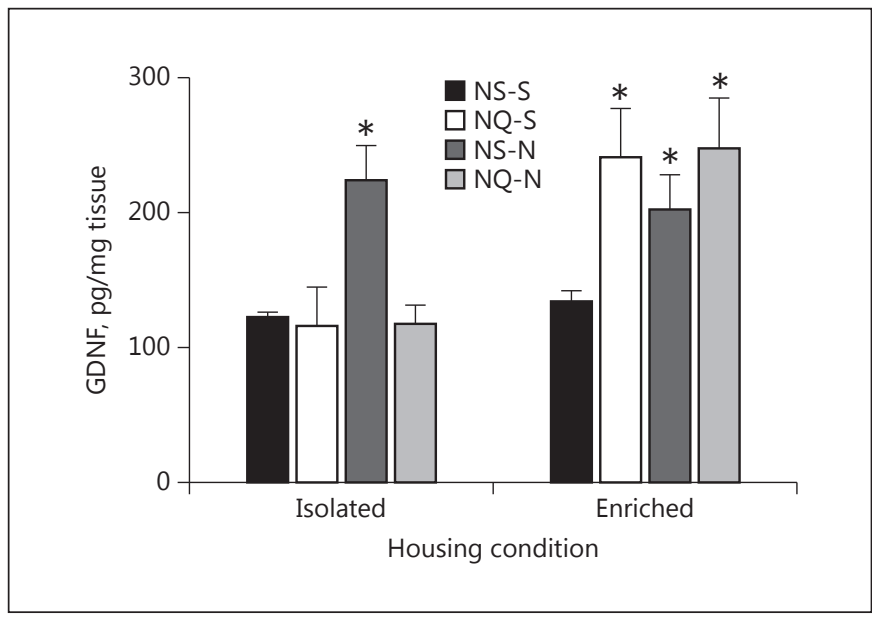

Fig. 2. Glial cell line-derived neurotrophic factor (GDNF) is presented as a function of neonatal drug treatment and housing condition. Isolated group NS-N was statistically equivalent to enriched groups NQ-S, NS-N, and NQ-N, all of which were statistically greater in GDNF protein levels compared to all other groups (indicated by ${ }^{*} p<0.05$ ).

ed animals sensitized to nicotine, because enriched group NS-N was equivalent to isolated group NS-N. Clearly, the interaction of housing condition, increases of dopamine $\mathrm{D}_{2}$ receptor sensitivity, and adolescent nicotine treatment is complex.

\section{Discussion}

This study demonstrated that environmental enrichment and isolated housing conditions during adolescence produced differential effects across neonatal drug treatment conditions in the NQ rodent model of psychosis. First, rats raised in the isolated condition demonstrated hyperactivity during habituation compared to animals raised in the enriched condition, consistent with past work demonstrating that environmental enrichment decreases open field locomotor activity and enhances the rate of habituation compared to isolated housing conditions $[38,39]$. Regarding the acute response to nicotine, all animals administered nicotine demonstrated a hypoactive response compared to isolated or enriched controls administered saline. Green et al. [40] reported that rats housed in an enriched environment demonstrated a more robust hypoactive response to nicotine compared to rats that were housed in isolation. However, this effect was observed to a much lower dose than was used in the pres-
68

Dev Neurosci 2018;40:64-72 DOI: $10.1159 / 000486391$
Brown/Schlitt/Owens/DePreter/ Cummins/Kirby/Gill/Burgess 
ent study ( 0.2 vs. $0.5 \mathrm{mg} / \mathrm{kg}$ base), and the animals tested were slightly older than the animals tested in the present study (P38 vs. P51). This age difference may seem trivial, but Andersen and colleagues $[35,41]$ have reported robust changes in the development of the dopamine system during adolescence and its response to psychostimulants, which may have impacted the results of the present study and that of Green et al. [40]. Further, the enhanced hypoactive effects observed in enriched rats in the latter study [40] was eliminated by a higher dose $(0.8 \mathrm{mg} / \mathrm{kg}$ base). Undoubtedly, the overall behavioral response to nicotine is dose dependent and enrichment may influence plasticity of the dopamine system during a critical period of development, which may underlie these differences between studies - NQ treatment enhanced sensitization to nicotine compared to NS treatment in enriched rats. In fact, enriched and isolated rats given NQ treatment were equivalent, and it does not appear that isolation during adolescence affected sensitization to nicotine if animals were administered NQ treatment. Further, isolated NStreated animals demonstrated sensitization to nicotine, whereas enrichment blocked sensitization to nicotine in NS-treated rats - somewhat similar to past work that demonstrated that enrichment attenuated nicotine sensitization [16] as well as amphetamine sensitization [42].

Regarding GDNF in the NAcc, the effects of housing conditions were also differential depending on neonatal drug treatment, but they did not necessarily echo behavioral effects. Enrichment robustly enhanced GDNF in NQ-treated rats, whereas there were no differences between the two housing conditions in NS-treated control rats (group NS-S). Interestingly, nicotine did not affect GDNF in NQ-treated rats. Regarding the effect of environmental enrichment, NQ-treated rats demonstrated increases in dopamine $\mathrm{D}_{2}$ sensitivity, and environmental enrichment has been shown to increase activity in dopaminergic neurons $[43,44]$, which results in an overall increase of GDNF. Interestingly, nicotine behavioral sensitization did result in a significant increase in accumbal GDNF of NS-treated rats regardless of housing conditions, but housing condition did not appear to affect GDNF in nicotine-treated rats. Therefore, nicotine may interact with environmental enrichment or the stress of isolated housing to ultimately result in an increase of GDNF in the NAcc. Regarding neurotrophic factors in general, there is evidence that, for example, BDNF increases in response to stress in the NAcc [45]. However, there are no data on how enrichment may interact with stress to affect GDNF. Regardless, in NS-treated controls, this increase of GDNF appears to be due to a combination

Effects of Environmental Enrichment on Nicotine Sensitization in NQ Rats of experience across differential housing conditions and the response to nicotine during behavioral sensitization.

There is a continually growing body of literature on the effects of environmental enrichment on the behavioral response to psychostimulants. Environmental enrichment has been shown to decrease the activating effects of psychostimulants $[46,47]$ or reduce self-administration of psychostimulants $[48,49]$ as well as natural rewards, such as sucrose [50]. Regarding behavioral sensitization to nicotine, environmental enrichment failed to alter sensitization to nicotine in NQ-treated rats, which were equivalent to isolated NQ rats also sensitized to nicotine. This particular effect is somewhat similar to findings with amphetamine in the poly I:C model of schizophrenia, in that environmental enrichment did not appear to alter exploratory behavior in rats that were administered poly I:C during gestation, nor change their behavioral response to amphetamine [31]. Especially relevant to the present study, Buschert et al. [31] found that neither environmental enrichment nor isolated housing conditions affected increased dopamine $\mathrm{D}_{2}$ receptor activation that is present in the poly I:C model. Therefore, it appears that environmental enrichment did not appear to alter the effects of nicotine in either the poly I:C or NQ rodent models of schizophrenia.

Overall, the behavioral findings in rodent models of psychosis relative to environmental enrichment are equivocal, dependent upon the model used and the behaviors analyzed. For example, $\mathrm{McOmish}$ et al. [25] demonstrated that environmental enrichment rescued loss of muscarinic cholinergic receptors in the neocortex and hippocampus of the PLC- $\beta_{1}$ knockout model in the mouse, but there were limited effects of enrichment on behavioral outcomes in this model, with no effect of environmental enrichment on prepulse inhibition, exploratory behavior, or cognitive deficits in the Morris water maze. Koseki et al. [26] found that environmental enrichment potentiated the hyperlocomotor behavioral response to acute treatment with phencyclidine, an antagonist to glutamatergic NMDA receptors which has relevance to the NMDA receptor hypofunction known to exist in schizophrenia [51]. Environmental enrichment also prevented chronic phencyclidine treatment-induced impairments of social behavior and recognition memory in the social interaction and novel object recognition tests, but neither sensitization nor associative effects of phencyclidine were analyzed in this study. Similar to this finding, environmental enrichment given during development appeared to reverse the sensorimotor gating impairment produced by neonatal treatment with MK-801,

Dev Neurosci 2018;40:64-72 DOI: $10.1159 / 000486391$ 
another known NMDA antagonist [27]. In addition, Santos et al. [29] reported that enrichment reduced behavioral deficits in the SHR compared to controls, and SHR have been shown to have alterations in glutamatergic receptor sensitivity [52]. Thus, it appears that environmental enrichment may be more effective with models centered around NMDA receptor manipulations, whereas enrichment does not appear to affect models that are known to manipulate the dopamine $\mathrm{D}_{2}$ receptor, another important hallmark of schizophrenia.

GDNF results revealed that NQ-treated rats housed in enrichment demonstrated a two-fold (100\%) increase in accumbal GDNF compared to NQ-treated rats housed in isolation. Interestingly, nicotine did not synergistically increase the accumbal GDNF response with environmental enrichment. However, nicotine resulted in a significant increase in GDNF of NS-treated rats, which was not affected by housing condition. One weakness of the current study is that we did not include animals that were socially housed, but all of our past work in the NQ model has been in socially housed rats. For example, in recent work [53], we reported that socially housed animals conditioned to nicotine in the CPP behavioral paradigm administered a slightly higher dose $(0.6 \mathrm{vs.} 0.5 \mathrm{mg} / \mathrm{kg}$ free base used in the present study) did not produce an increase of GDNF in the NAcc, although there were increases in NAcc GDNF in NQ-treated rats given nicotine, similar to the effects observed here. In the current study, it appears that enrichment has a particularly robust effect on accumbal GDNF in NQ-treated animals that does not appear to be influenced by nicotine, whereas nicotine may interact with housing condition in a presumably different way to influence accumbal GDNF in NS-treated rats. This suggests that there is a very different interaction between experience and increases in dopamine $\mathrm{D}_{2}$ sensitivity as is produced by NQ treatment, which adds to the literature of the interaction of environment and behavior in rodent models of psychosis [for review, see 54]. However, in past work we have analyzed the effects of nicotine extensively in the NQ model, and generally found enhanced activating effects to nicotine $[55,56]$ as well as enhanced responses to BDNF [55]. Based on the data here, it appears that GDNF certainly interacts with experience in different ways depending on the changes in the dopamine system produced by neonatal drug treatment. Future studies will analyze further mechanisms, especially related to neural plasticity, regarding the interaction of environmental experience with the NQ model and the behavioral response to nicotine.

It is clear from these data that environmental experience has an important influence on the response to nicotine in a rodent model of schizophrenia, and this is the first study to analyze this effect. These results are especially important in the context of increased smoking behavior in individuals diagnosed with schizophrenia and the poor health outcome that is produced [56]. This study demonstrated that environmental enrichment did not influence behavioral sensitization to nicotine compared to isolation rearing during the adolescent period in the NQ rodent model of dopamine $\mathrm{D}_{2}$ supersensitivity, a rodent model which has relevance to schizophrenia. This has implications towards the limitations of experiential manipulations in rodent models of psychosis and was similar to findings with the poly I:C model [31]. However, environmental enrichment did influence accumbal GDNF protein in NQ rats, although nicotine did not interact with that effect and, interestingly, nicotine had a much more prominent influence on GDNF in controls. Although environmental enrichment was employed only during the adolescent period in the present study, future work may focus on these same manipulations in the behavioral response to nicotine in other behavioral tasks, such as conditioned place preference or nicotine self-administration, and the underlying mechanisms of these effects.

\section{Acknowledgments}

The authors would like to thank James D. Wherry for help in completing this project. This project was supported by NIH grant 1R15DA034912-A1 (R.W.B.).

\section{Disclosure Statement}

The authors declare no conflicts of interest.

References
Brown/Schlitt/Owens/DePreter/ Cummins/Kirby/Gill/Burgess 
4 Perna MK, Brown RW: Adolescent nicotine sensitization and effects of nicotine on accumbal dopamine release in a rodent model of increased dopamine $\mathrm{D}_{2}$ receptor sensitivity. Behav Brain Res 2013;242:102-109.

5 Cope ZA, Huggins KN, Sheppard AB, Noel DM, Roane DS, Brown RW: Neonatal quinpirole treatment enhances locomotor activation and dopamine release in the nucleus accumbens core in response to amphetamine treatment in adulthood. Synapse 2010;64: 289-300.

6 Nowak P, Brus R, Kostrzewa RM: Amphetamine-induced enhancement of neostriatal in vivo microdialysate dopamine content in rats, quinpirole-primed as neonates. Pol J Pharmacol 2001;53:319-29.

7 Brown RW, Maple AM, Perna MK, Sheppard AB, Cope ZA, Kostrzewa RM: Schizophrenia and substance abuse comorbidity: nicotine addiction and the neonatal quinpirole model. Dev Neurosci 2012;34:140-51.

8 Monteiro P, Feng G: Learning from animal models of obsessive-compulsive disorder. Biol Psychiatry 2016;79:7-16.

9 Grant BF, Hasin DS, Chou SP, Stinson FS, Dawson DA. Nicotine dependence and psychiatric disorders in the United States: results from the national epidemiologic survey on alcohol and related conditions. Arch Gen Psychiatry 2004;61:1107-1115.

10 de Leon J, Diaz FJ: A meta-analysis of worldwide studies demonstrates an association between schizophrenia and tobacco smoking behaviors. Schizophr Res 2005;76:135-157.

11 Lipari RN, Hughes A: State Estimates of Adolescent Cigarette Use and Perceptions of Risk of Smoking: 2012 and 2013. The CBHSQ Report. Rockville, Substance Abuse and Mental Health Services Administration, 2013.

12 Pierce RC, Kalivas PW: A circuitry model of the expression of behavioral sensitization to amphetamine-like psychostimulants. Brain Res Rev 1997;25:192-216.

13 Shim I, Javaid JI, Wirtshafter D, Jang SY, Shin KH, Lee HJ, Chung YC, Chun BG: Nicotineinduced behavioral sensitization is associated with extracellular dopamine release and expression of c-Fos in the striatum and nucleus accumbens of the rat. Behav Brain Res 2001; 121:137-147.

14 Bradberry CW: Cocaine sensitization and dopamine mediation of cue effects in rodents, monkeys, and humans: areas of agreement, disagreement, and implications for addiction. Psychopharmacology 2007;191:705-717.

15 Gipson CD, Beckmann JS, El-Maraghi S, Marusich JA, Bardo MT: Effect of environmental enrichment on escalation of cocaine self-administration in rats. Psychopharmacology 2011;214:557-566.

16 Hamilton KR, Elliott BM, Berger SS, Grunberg, NE: Environmental enrichment attenuates nicotine behavioral sensitization in male and female rats. Exp Clin Psychopharmacol 2014;22:356-363.
17 Meyer AC, Bardo MT: Amphetamine self-administration and dopamine function: assessment of gene $\times$ environment interactions in Lewis and Fischer 344 rats. Psychopharmacology 2015;232:2275-2285.

18 Ding Y, Lin K, Li B, Ma L: Enhanced cocaine self-administration in adult rats with adolescent isolation experience. Pharmacol Biochem Behav 2005;82:673-677.

19 Cain ME, Mersmann MG, Gill MJ, Pittenger ST: Dose-dependent effects of differential rearing on amphetamine-induced hyperactivity. Behav Pharmacol 2012;23:744-753.

20 Pham TM, Ickes B, Albeck D, Söderström S, Granholm AC, Mohammed AH: Changes in brain nerve growth factor levels and nerve growth factor receptors in rats exposed to environmental enrichment for one year. Neuroscience 1999;94:279-286.

21 Ickes BR, Pham TM, Sanders LA, Albeck DS, Mohammed AH, Granholm AC: Long-term environmental enrichment leads to regional increases in neurotrophin levels in rat brain. Exp Neurol 2000;164:45-52.

22 Wang J, Carnicella S, Ahmadiantehrani S, He DY, Barak S, Kharazia V, Ben Hamida S, Zapata A, Shippenberg TS, Ron D: Nucleus accumbens-derived glial cell line-derived neurotrophic factor is a retrograde enhancer of dopaminergic tone in the mesocorticolimbic system. J Neurosci 2010;30:14502-14512.

23 Young D, Lawlor PA, Leone P, Dragunow M, During MJ: Environmental enrichment inhibits spontaneous apoptosis, prevents seizures and is neuroprotective. Nat Med 1999; 5:448-453.

24 Fone KC, Porkess MV: Behavioural and neurochemical effects of post-weaning social isolation in rodents - relevance to developmental neuropsychiatric disorders. Neurosci Biobehav Rev 2008;32:1087-1102.

25 McOmish CE, Burrows E, Howard M, Scarr E, Kim D, Shin HS, Dean B, van den Buuse M, Hannan AJ: Phospholipase C- $\beta 1$ knockout mice exhibit endophenotypes modeling schizophrenia which are rescued by environmental enrichment and clozapine administration. Mol Psychiatry 2008;13:661-672.

26 Koseki T, Mouri A, Mamiya T, Aoyama Y, Toriumi K, Suzuki S, Nakajima A, Yamada T, Nagai T, Nabeshima T: Exposure to enriched environments during adolescence prevents abnormal behaviours associated with histone deacetylation in phencyclidine-treated mice. Int J Neuropsychopharmacol 2012;15:14891501.

27 Nozari M, Shabani M, Hadadi M, Atapour N: Enriched environment prevents cognitive and motor deficits associated with postnatal MK-801 treatment. Psychopharmacology 2014;231:4361-4370.

28 Melik E, Babar E, Kocahan S, Guven M, Akillioglu K: Enriched environment has limited capacity for the correction of hippocampal memory-dependent schizoid behaviors in rats with early postnatal NMDAR dysfunction. Int J Dev Neurosci 2014;33:22-28.
29 Santos CM, Peres FF, Diana MC, Justi V, Suiama MA, Santana MG, Abílio VC: Peripubertal exposure to environmental enrichment prevents schizophrenia-like behaviors in the SHR strain animal model. Schizophr Res 2016;176:552-559.

30 Reisinger S, Khan D, Kong E, Berger A, Pollak A, Pollak DD: The poly(I:C)-induced maternal immune activation model in preclinical neuropsychiatric drug discovery. Pharmacol Ther 2015;149:213-226.

31 Buschert J, Sakalem ME, Saffari R, Hohoff C, Rothermundt M, Arolt V, Zhang W, Ambrée O: Prenatal immune activation in mice blocks the effects of environmental enrichment on exploratory behavior and microglia density. Prog Neuropsychopharmacol Biol Psychiatry 2016;67:10-20.

32 Mao D, McGehee DS: Nicotine and behavioral sensitization. J Mol Neurosci 2010;40:154163.

33 Maple AM, Smith KJ, Perna MK, Brown RW: Neonatal quinpirole treatment produces prepulse inhibition deficits in adult male and female rats. Pharm Biochem Behav 2015;137: 93-100.

34 Rahman Z, Schwarz J, Gold SJ, Zachariou V Wein MN, Choi KH, Kovoor A, Chen CK, DiLeone RJ, Schwarz SC, Selley DE, Sim-Selley LJ, Barrot M, Luedtke RR, Self D, Neve RL, Lester HA, Simon MI, Nestler EJ: RGS9 modulates dopamine signaling in the basal ganglia. Neuron 2003;38:941-52.

35 Andersen SL, Teicher MH: Sex differences in dopamine receptors and their relevance to ADHD. Neurosci Biobehav Rev 2000;24:137141.

36 Davis J, Eyre H, Jacka FN, Dodd S, Dean O, McEwen S, Debnath M, McGrath J, Maes M, Amminger P, McGorry PD, Pantelis C, Berk M: A review of vulnerability and risks for schizophrenia: beyond the two hit hypothesis. Neurosci Biobehav Rev 2016;65:185-194.

37 Hornbeck P: Assays for antibody production; in Colligan JE, Kruisbeek AM, Margulies DH (eds): Current Protocols in Immunology. New York, Wiley, 1994, pp 2.1.1-2.1.22.

38 Varty GB, Paulus MP, Braff DL, Geyer MA: Environmental enrichment and isolation rearing in the rat: effects on locomotor behavior and startle response plasticity. Biol Psychiatry 2000;47:864-873.

39 Hughes RN, Collins MA: Enhanced habituation and decreased anxiety by environmental enrichment and possible attenuation of these effects by chronic alpha-tocopherol (vitamin E) in aging male and female rats. Pharmacol Biochem Behav 2010;94:534-542.

40 Green TA, Cain ME, Thompson M, Bardo MT: Environmental enrichment decreases nicotine-induced hyperactivity in rats. Psychopharmacology 2003;170:235-241.

41 Chen YI, Choi JK, Xu H, Ren J, Andersen SL, Jenkins BG: Pharmacologic neuroimaging of the ontogeny of dopamine receptor function. Dev Neurosci 2010;32:125-138. 
42 Adams E, Klug J, Quast M, Stairs DJ: Effects of environmental enrichment on nicotine-induced sensitization and cross-sensitization to D-amphetamine in rats. Drug Alcohol Depend 2013;129:247-253.

43 Lobo MK, Zaman S, Damez-Werno DM, Koo JW, Bagot RC, DiNieri JA, Nugent A, Finkel E, Chaudhury D, Chandra R, Riberio E, Rahkin J, Mouzon E, Cachope R, Cheer, JF, Han MH, Dietz DM, Self DW, Hurd YL, Vialou V, Nestler EJ: $\Delta$ FosB induction in striatal medium spiny neuron subtypes in response to chronic pharmacological, emotional, and optogenetic stimuli. J Neurosci 2013;33:1838118395.

44 Thiriet N, Amar L, Toussay X, Lardeux V, Ladenheim B, Becker KG, Cadet JL, Solinas $\mathrm{M}$, Jaber M: Environmental enrichment during adolescence regulates gene expression in the striatum of mice. Brain Res 2008; 1222 : 31-41.

45 Berton O, McClung CA, Dileone RJ, Krishnan V, Renthal W, Russo SJ, Graham D, Tsankova NM, Bolanos CA, Rios M, Monteggia LM, Self DW, Nestler EJ: Essential role of BDNF in the mesolimbic dopamine pathway in social defeat stress. Science 2006;311:864868 .
46 Bowling SL, Rowlett JK, Bardo MT: The effect of environmental enrichment on amphetamine-stimulated locomotor activity, dopamine synthesis and dopamine release. Neuropharmacology 1993;32:885-893.

47 Solinas M, Chauvet C, Thiriet N, El Rawas R Jaber M: Reversal of cocaine addiction by environmental enrichment. Proc Natl Acad Sci USA 2008;105:17145-17150.

48 Bardo MT, Klebaur JE, Valone JM, Deaton C: Environmental enrichment decreases intravenous self-administration of amphetamine in female and male rats. Psychopharmacology 2001;155:278-284

49 Venebra-Muñoz A, Corona-Morales A, Santiago-García J, Melgarejo-Gutiérrez M, Caba M, García-García F: Enriched environment attenuates nicotine self-administration and induces changes in $\Delta \mathrm{FosB}$ expression in the rat prefrontal cortex and nucleus accumbens. Neuroreport 2014;25:688-692.

50 Grimm JW, Osincup D, Wells B, Manaois M, Fyall A, Buse C, Harkness JH: Environmental enrichment attenuates cue-induced reinstatement of sucrose seeking in rats. Behav Pharmacol 2008;19:777-785.

51 Yamamoto H, Hagino Y, Kasai S, Ikeda K: Specific roles of NMDA receptor subunits in mental disorders. Curr Mol Med 2015;15: 193-205.
52 Jensen V, Rinholm JE, Johansen TJ, Medin T, Storm-Mathisen J, Sagvolden T,Hvalby O, Bergersen LH: N-methyl-D-aspartate receptor subunit dysfunction at hippocampal glutamatergic synapses in an animal model of attention-deficit/hyperactivity disorder. Neuroscience 2009;158:353-364.

53 Brown RW, Kirby SL, Denton AR, Dose JM, Cummins ED, Drew Gill W, Burgess KC: An analysis of the rewarding and aversive associative properties of nicotine in the neonatal quinpirole model: effects on glial cell line-derived neurotrophic factor (GDNF). Schizophr Res 2017, DOI: 10.1016/j.schres.2017.03.024.

54 Burrows EL, Hannan AJ: Cognitive endophenotypes, gene-environment interactions and experience-dependent plasticity in animal models of schizophrenia. Biol Psychol 2016; 116:82-89.

55 Perna MK, Brown RW: Adolescent nicotine sensitization and effects of nicotine on accumbal dopamine release in a rodent model of increased dopamine $\mathrm{D}_{2}$ receptor sensitivity. Behav Brain Res 2013;242:102-109.

56 Perna MK, Cope ZA, Maple AM, Longacre ID, Correll JA, Brown RW: Nicotine sensitization in adult male and female rats quinpirole-primed as neonates. Psychopharmacology 2008;199:67-75. 